\title{
Identification of a complete sample of northern ROSAT All-Sky Survey X-ray sources
}

\section{The optical observations}

\author{
F.-J. Zickgraf ${ }^{\star}, 1,2$, I. Thiering ${ }^{1}$, J. Krautter ${ }^{1}$, I. Appenzeller ${ }^{1}$, R. Kneer ${ }^{1}$, W.H. Voges $^{2}$, B. Ziegler ${ }^{1,5}$, \\ C. Chavarria ${ }^{6}$, A. Serrano ${ }^{3}$, R. Mujica ${ }^{3}$, M. Pakull ${ }^{4}$, and J. Heidt ${ }^{1}$ \\ 1 Landessternwarte Königstuhl (LSW), D-69117 Heidelberg, Germany \\ 2 Max-Planck-Institut für Extraterrestrische Physik (MPE), Giessenbachstrasse, Postfach 1603, D-85740 Garching, Germany \\ 3 Instituto Nacional de Astrofisica, Optica y Electronica (INAOE), A. Postal 51 y 216 Z.P., 72000 Puebla, Mexico \\ 4 Observatoire de Strasbourg, 11 rue de l'Université, F-67000 Strasbourg, France \\ 5 Universitätssternwarte München, Scheinerstr. 1, D-81679 München, Germany \\ 6 Instituto de Astronomia-UNAM, Apdo Postal 70-264, 04510 México D.F., Mexico
}

Received August 29; accepted September 5, 1996

\begin{abstract}
We describe a program for the optical identification of a representative sample of RASS sources contained in six study areas north of $\delta=-9^{\circ}$, with $\left|b^{\mathrm{II}}\right| \gtrsim 20^{\circ}$ including one region near the North Galactic pole (NGP) and one near the North Ecliptic pole (NEP) and present some preliminary results. A full identification has been carried out for a count-rate and area-limited complete subsample comprising 674 sources. The ratio of "extragalactic" ( e.g. AGN, clusters of galaxies) to "stellar" (e.g. coronal emitters, active binaries) counterparts was found to depend on the galactic neutral hydrogen column density, $N_{\mathrm{H}}$. In the area near the NGP (low $N_{\mathrm{H}}$ ) "extragalactic" counterparts dominate, while in the area with the highest $N_{\mathrm{H}}$ most X-ray sources are identified with stellar counterparts.
\end{abstract}

Key words: surveys - X-rays: general - X-rays: galaxies - X-rays: stars - galaxies: active - galaxies: cluster: general

\section{Introduction}

During the ROSAT All-Sky Survey (RASS) about 60000 $\mathrm{X}$-ray sources were detected in the soft X-ray range between 0.1 and $2.4 \mathrm{keV}$ with the PSPC detector (Voges

Send offprint requests to: F.-J. Zickgraf

* Present address: Max-Planck-Institut für Extraterrestrische Physik, Giessenbachstr., Postfach 1603, 85740 Garching, Germany.
1994). This large number of sources does not allow a complete identification of all optical counterparts. However, a statistical classification of the RASS source content can be obtained from much smaller complete subsamples if variations of the column density of neutral hydrogen, $N_{\mathrm{H}}$, the background, and the integration time are taken into account. Such complete samples of X-ray sources are obviously an important and valuable basis for the study of $\mathrm{X}$-ray properties of AGN, galaxy clusters, and coronal and other galactic X-ray emitters. At low galactic latitudes the RASS is investigated in this way by the ROSAT Galactic Plane Survey (e.g. Motch et al. 1991). Our objective is the investigation of the RASS at high galactic latitudes $\left(\left|b^{\mathrm{II}}\right| \gtrsim 20^{\circ}\right)$.

Our knowledge of the composition of the X-ray sky at high galactic latitude is based to a large extent on the results of the EINSTEIN Medium Sensitivity Survey (EMSS) (Gioia et al. 1990; Stocke et al. 1991). A basic result was that $51 \%$ of the EMSS sources are AGN (QSOs, Seyferts) and $26 \%$ are of stellar origin. The remaining $23 \%$ are BL Lacs (4\%), normal and cooling flow galaxies $(3 \%)$, clusters of galaxies (12\%), and unidentified sources (4\%). Recently, deep pointed ROSAT surveys such as the Lockman Hole studied by Hasinger et al. (1993), (1994) and the QSF3 field by Shanks et al. (1991) contributed new information, in particular concerning the composition of the soft X-ray background. In the deep sample studied by Hasinger et al. more than $60 \%$ of the sources were found to be AGN whereas the stellar sources contribute only $8 \%$. Shanks et al. obtained a similar result.

In the RASS we can expect a composition different from these samples: The sources in the EMSS were found serendipitously in fields of otherwise "interesting" 
objects. Therefore this survey is not representing the average X-ray sky with respect to the background and, in particular, $N_{\mathrm{H}}$ which could introduce a bias in the composition of the sample. If e.g. $N_{\mathrm{H}}$ in the locations of the EMSS targets is lower than on the average in the RASS at high galactic latitude, the sample would be biased towards extragalactic sources. Furthermore, compared to ROSAT the EMSS energy band is harder. Therefore, in the EMSS preferentially harder X-ray sources were selected. Differences in the relative fractions of the various object classes due to the energy bands are e.g. suggested by the results of the EXOSAT High Galactic Latitude Survey (HGLS) (Giommi et al. 1991) which covered an energy band very similar to that of ROSAT $(0.05-2 \mathrm{keV})$. This survey contained a significantly higher fraction of stellar counterparts than the EMSS. In the deep ROSAT surveys quoted above the detected sources are mostly at $\mathrm{X}$-ray fluxes one to two order of magnitude below the flux limit in the RASS. In addition, the column density of neutral hydrogen is generally low in the directions of the deep surveys (e.g. the Lockman Hole) and the area of sky covered is quite small.

Therefore we started an optical identification program for northern RASS X-ray sources at high galactic latitude. The goal was to identify optical counterparts of all X-ray sources in the selected sample. This investigation is a collaboration of the Landessternwarte (LSW), Heidelberg, the Max-Planck-Institut für Extraterrestrische Physik (MPE), Garching, and the Instituto Nacional de Astrofisica, Optica y Electronica (INAOE), Puebla. A similar investigation is being carried out in the southern hemisphere as an ESO key program (Danziger et al.)

Details of the X-ray survey data and their reduction have been discussed in Voges et al. (in preparation, Paper I). The full catalog of the identifications is published in Thiering et al. (in preparation, Paper III). A detailed statistical analysis of the results is given by Krautter et al. (in preparation, Paper IV). In the present paper we describe the methods of the optical identifications and we discuss first results. In Sect. 2 the RASS sample is discussed. The optical observations and the identification procedure are described in Sects. 3 and 4. In Sect. 5 preliminary results are presented.

\section{The RASS sample}

For our investigation originally six "study areas" north of $\delta=-9^{\circ}$, designated I to VI, have been selected. Each area has a size of about 144 square degrees. One of the areas chosen is located close to the North Ecliptic pole (NEP), area $\mathrm{V}$, a second one around the North Galactic pole (NGP), area IV. The other four areas are in regions of medium-to-high X-ray sensitivity outside the galactic plane $\left(\left|b^{\mathrm{II}}\right| \gtrsim 20^{\circ}\right)$ at R.A.s allowing year-round follow-up observations. The coordinates of the study areas are given in Table 1.
Table 1. Coordinates (right ascension R.A.(2000.0) and declination DEC(2000.0)) of the selected study areas. Areas IVac and $\mathrm{Va}$ are subsections of areas IV and V, respectively (see Sect. 2.1). In the last column the total number of RASS sources with a detection likelihood of $\geq 10$ is given for each study area

\begin{tabular}{llll}
\hline study area & \multicolumn{2}{c}{ area boundaries } & number \\
& R.A. & R.A. & of sources \\
& DEC & DEC & \\
\hline area I & $03^{\mathrm{h}} 28.0^{\mathrm{m}}$ & $04^{\mathrm{h}} 18.0^{\mathrm{m}}$ & 147 \\
& $-03^{\circ}$ & $+09^{\circ}$ & \\
area II & $07^{\mathrm{h}} 02.0^{\mathrm{m}}$ & $08^{\mathrm{h}} 38.0^{\mathrm{m}}$ & 178 \\
& $+57^{\circ}$ & $+63^{\circ}$ & \\
& $06^{\mathrm{h}} 34.0^{\mathrm{m}}$ & $08^{\mathrm{h}} 29.0^{\mathrm{m}}$ & \\
& $+63^{\circ}$ & $+69^{\circ}$ & \\
area III & $10^{\mathrm{h}} 16.0^{\mathrm{m}}$ & $11^{\mathrm{h}} 06.0^{\mathrm{m}}$ & 188 \\
& $-09^{\circ}$ & $+03^{\circ}$ & \\
area IV & $11^{\mathrm{h}} 57.6^{\mathrm{m}}$ & $12^{\mathrm{h}} 55.8^{\mathrm{m}}$ & 317 \\
& $+27^{\circ}$ & $+39^{\circ}$ & \\
area IVac & $11^{\mathrm{h}} 57.6^{\mathrm{m}}$ & $12^{\mathrm{h}} 26.0^{\mathrm{m}}$ & 137 \\
& $+27^{\circ}$ & $+39^{\circ}$ & \\
area V & $17^{\mathrm{h}} 19.8^{\mathrm{m}}$ & $19^{\mathrm{h}} 19.8^{\mathrm{m}}$ & 671 \\
& $+75^{\circ}$ & $+81^{\circ}$ & \\
& $16^{\mathrm{h}} 17.0^{\mathrm{m}}$ & $19^{\mathrm{h}} 57.0^{\mathrm{m}}$ & \\
& $+69^{\circ}$ & $+75^{\circ}$ & \\
area Va & $16^{\mathrm{h}} 17.0^{\mathrm{m}}$ & $17^{\mathrm{h}} 37.4^{\mathrm{m}}$ & 183 \\
& $+69^{\circ}$ & $+75^{\circ}$ & \\
area VI & $21^{\mathrm{h}} 52.0^{\mathrm{m}}$ & $22^{\mathrm{h}} 42.0^{\mathrm{m}}$ & 128 \\
& $+03^{\circ}$ & $+15^{\circ}$ & \\
& & &
\end{tabular}

As described in detail in Paper I, the individual RASS scan stripes covering our study areas were merged to produce a final data set. Then sources were identified by applying standard maximum-likelihood search algorithms. For the source detection a minimum detection likelihood $L$ of 10 was adopted corresponding to detections on a $4 \sigma$ level, where $L=-\ln P$ with $1-P$ being the probability of a spurious detection. This detection limit was reached for count rates of $\sim 0.003 \mathrm{cts} \mathrm{s}^{-1}$ in area $V$ and $\sim 0.01 \mathrm{cts} \mathrm{s}^{-1}$ in all other areas. The final data set of the six study areas comprises 1629 X-ray sources for which positions, integrated X-ray count rates $C R$ in the 0.1 to $2.4 \mathrm{keV}$ energy band, hardness ratios $H R 1$ and $H R 2$, extension parameter $E X T$, and detection likelihoods are available.

The hardness ratios are defined as

$H R 1=\frac{[B]-[A]}{[B]+[A]}$

and

$H R 2=\frac{[D]-[C]}{[D]+[C]}$,

where $[A]$, etc. are the count rates in the respective energy bands $A=0.11-0.41 \mathrm{keV}, B=0.52-2.01 \mathrm{keV}$, $C=0.52-0.90 \mathrm{keV}$, and $D=0.91-2.01 \mathrm{keV}$.

The extension parameter EXT gives a measure for the deviation of the source shape from a point source and can thus be used to identify extended sources like clusters of galaxies. 


\subsection{The count-rate and area limited complete subsample}

After the superposition of the scan stripes the total number of sources detected in the originally selected study areas was larger than anticipated and also larger than required for the statistical investigation. Moreover, the different sensitivity resulted in very different source numbers in the original study areas.

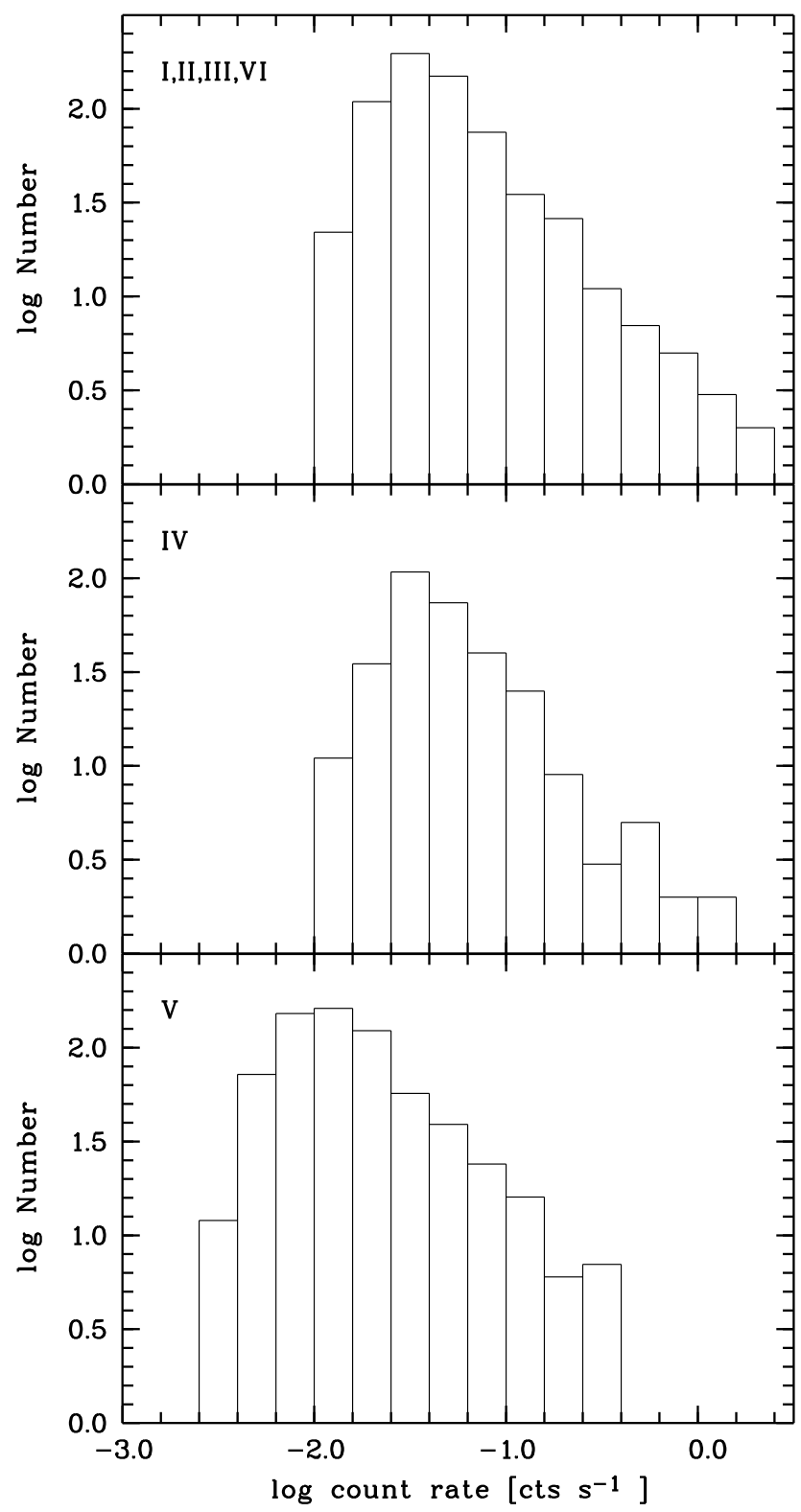

Fig. 1. Number of the detected sources as a function of the count rate for the combined study areas I, II, III, and VI (upper panel) and for areas IV and V (two lower panels). Incompleteness starts below $0.01 \mathrm{cts} \mathrm{s}^{-1}$ in area $\mathrm{V}$ and below $0.03 \mathrm{cts} \mathrm{s}^{-1}$ in the other areas
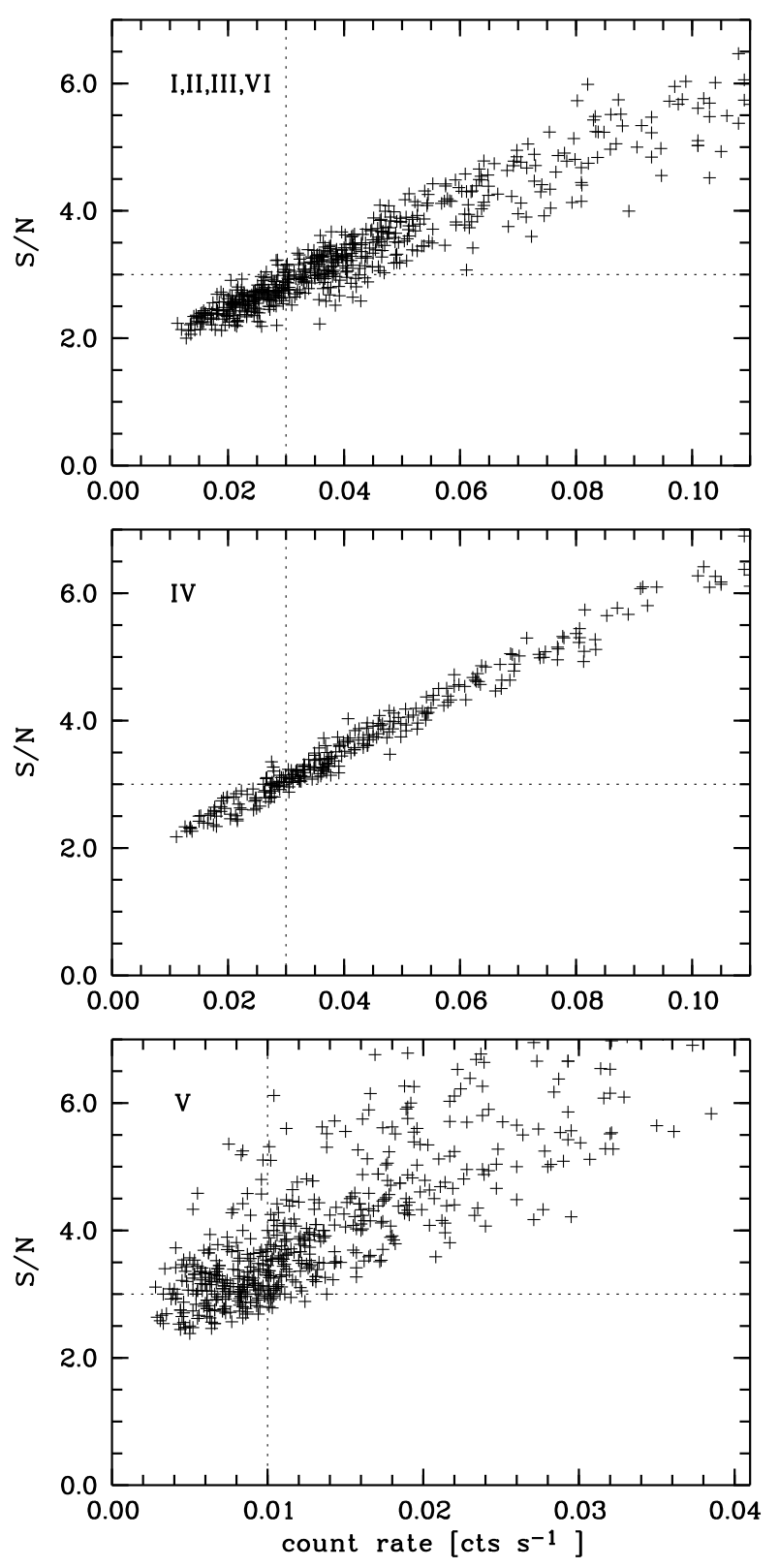

Fig. 2. Signal-to-noise ratio $(S / N)$ of the X-ray fluxes in the six study areas I to VI as a function of the X-ray count rates. The broken vertical line indicates the flux limits adopted for our complete sample. Note the different behaviour of the NEP field $\mathrm{V}$, where typical integration times were higher and less uniform

To reduce the sample to a manageable but still statistically meaningful size, we removed all sources below a minimum count rate. These minimum count rates were selected using histograms of $\log$ (source number) over $\log (C R)$. As can be seen in Fig. 1, the X-ray sample becomes incomplete at count rates lower than about $0.01 \mathrm{cts} \mathrm{s}^{-1}$ in area $\mathrm{V}$, and about $0.03 \mathrm{cts} \mathrm{s}^{-1}$ in the other areas. For the countrate limits adopted the $\mathrm{S} / \mathrm{N}$ ratio of the $\mathrm{X}$-ray fluxes is equal to or larger than about 3 (see Fig. 2). 
In addition, we decreased the sample further by restricting the condition of complete identification in the NGP and NEP areas to smaller sub-areas. They are designated IVac and Va in Table 1. Area IVac contains the western half of area IV with count rates $\geq 0.03 \mathrm{ctss}^{-1}$. During the course of the project, area Va which consists of the sources in the western quarter of area $\mathrm{V}$ with count rates $\geq 0.01 \mathrm{cts} \mathrm{s}^{-1}$ was extended in R.A. towards the east until it finally resulted in a $6.2 \times 6^{\circ}$ field size. For areas I, II, III and VI we adopted count rate limits of $\geq$ $0.03 \mathrm{cts} \mathrm{s}^{-1}$ for the entire $12 \times 12^{\circ}$ areas. The so-defined final subsample contains 674 sources and the number of sources in each field is about equal. Table 2 summarizes basic informations about this subsample.

We note that the median column density of neutral hydrogen, $N_{\mathrm{H}}$, (taken from Dickey \& Lockman (1990) varies by a factor of about 7 between the different study areas (see Table 2) with a scatter of about a factor of $\lesssim 2$ within each area. In area I the scatter is larger with $N_{\mathrm{H}}$ ranging between 6 and $1910^{20} \mathrm{~cm}^{-2}$. The median integration time in area Va is about 5 to 7 times longer than in the other areas resulting in a $2-3$ times higher signal-to-noise ratio for the same count-rate level.

While a complete identification was carried out only for the subsample defined by Table 2, our final catalog presented in Paper III will also contain identifications outside these areas but inside the areas defined by Table 1 .

\subsection{Optical brightness limits for the subsample}

For the optical identification it is helpful to obtain an estimate for the visual brightness of the faintest expected counterpart for the various object classes in the studied sample. The results of the EMSS (Stocke et al. 1991) have shown that different classes of X-ray emitters represent different rather narrow ranges in the $\mathrm{X}$-ray-to-optical flux ratios $f_{\mathrm{x}} / f_{V}$ with $f_{\mathrm{x}}$ and $f_{V}$ being the $\mathrm{X}$-ray and visual fluxes, respectively. The knowledge of $f_{\mathrm{x}} / f_{V}$ thus can help in the identification process by allowing to exclude or include objects of a certain visual brightness as possible counterparts. In order to estimate the optical brightness of the faintest expected counterparts we therefore made use of the flux ratios $\log f_{\mathrm{x}} / f_{V}$ from the EMSS. The visual flux, $f_{V}$, was calculated with the relation given by Stocke et al. (1991). Due to the energy bands of the detectors, RASS sources could have X-ray-to-optical flux ratios differing from those derived with EINSTEIN. For AGN an estimate for the difference $\Delta$ between EMSS $\log f_{\mathrm{x}} / f_{V}$ values and the expected corresponding ROSAT ratios can be obtained by assuming a power law energy distribution. For a photon index of $\Gamma=2$ as adopted below the difference $\Delta$ is on the order of $\sim-0.1$; for $\Gamma=2.5$ it is $\sim-0.3$. For coronal emitters Raymond-Smith models (Raymond \& Smith 1977) with temperatures of $0.2 \mathrm{keV}$ and $1 \mathrm{keV}$ (see below) lead to $\Delta \approx-0.2$ and -0.1 , respectively. This leads to differences of the visual magnitude limits of $\lesssim 0.5^{\mathrm{m}}$. For the estimates derived in the following we neglect effects due to the different energy bands of the RASS and of the EMSS.

The X-ray flux limits for our subsample are given by the applied count rate limits which have to be converted to fluxes. To obtain the conversion factor for coronal emitters we assumed a bremsstrahlung X-ray spectrum of a thermal plasma with metal absorption lines. It can be described by a Raymond-Smith model. Because the stellar sources are mostly nearby for our purpose the foreground absorption due to neutral hydrogen can be assumed to be similar in all study areas. Typical temperatures in stellar coronae are between $810^{5}$ to $210^{7} \mathrm{~K}$. Assuming a Raymond-Smith model then leads to an energy conversion factor, $E C F$, between count rate and X-ray flux of $E C F \approx 1.710^{11} \mathrm{cts} \mathrm{cm}^{2} \mathrm{erg}^{-1}$ (cf. also Schmitt et al. 1995) for the ECF of low-mass stars). With this conversion factor the X-ray flux limits are about $f_{\mathrm{x}}^{\text {cor }} \approx 610^{-14} \mathrm{erg} \mathrm{cm}^{-2}$ and $1.8^{-13} \mathrm{erg} \mathrm{cm}^{-2}$ in area Va and in the other areas, respectively.

X-ray flux limits for extragalactic sources depend on the shape of the spectrum and, in particular, on the column density of neutral hydrogen, $N_{\mathrm{H}}$, due to the dependence of the conversion factor on these quantities. For each study area the median value for $N_{\mathrm{H}}$ was taken from Dickey \& Lockman (1990). For AGN the flux limits given in Table 3 were obtained for a power law spectrum $f_{\nu} \propto \nu^{-\alpha}$ with $\alpha=1.0$ corresponding to a photon index $\Gamma=2.0$. For clusters of galaxies a thermal bremsstrahlung spectrum with $T=10^{8} \mathrm{~K}$ was assumed. Likewise, for normal galaxies thermal bremsstrahlung with $T=1.210^{7} \mathrm{~K}$ was assumed corresponding to a thermal energy of $k T \approx 1 \mathrm{keV}$ as derived by Kim et al. (1992) for ellipticals (cf. also Read et al. 1996 and Peace \& Sansom 1996). For spirals Kim et al. found $k T \approx 3 \mathrm{keV}$. We give the flux limits for ellipticals which generally have higher X-ray luminosities than spirals (see also Sect. 4.3).

The highest ratio $\log f_{\mathrm{x}} / f_{V}$ for coronal emitters was found for $M$ type stars (Stocke et al. 1991). With $\log f_{\mathrm{x}} / f_{V}=-3.1$ to -0.5 from the EMSS our flux limits correspond to $V_{\min } \lesssim 18.4^{\mathrm{m}}$ for area $V a$ and $17.2^{\mathrm{m}}$ for the other areas, respectively.

Assuming the upper limits of the EMSS $\log f_{\mathrm{x}} / f_{V}$ values for AGN the expected visual magnitude limits in the subsample are:

$V \approx 19.5^{\mathrm{m}}$ in area $\mathrm{I}$,

$V \approx 20^{\mathrm{m}}$ in areas II, III, and VI,

$V \approx 20.5^{\mathrm{m}}$ in area IVac,

$V \approx 21^{\mathrm{m}}$ in area Va,

with a scatter on the order of $0.5^{\mathrm{m}}$ due to the variations of $N_{\mathrm{H}}$ within the individual study areas. AGN with $V$ as faint as $20^{\mathrm{m}}$ to $21^{\mathrm{m}}$ could be identified despite the low exposure levels in the continuum by means of their emission lines. A problem are the X-ray bright but optically faint BL Lac objects. Due to their $\log f_{\mathrm{x}} / f_{V}$ values they can be as faint as $21^{\mathrm{m}}$ in area Va. With the $2.15 \mathrm{~m}$ telescope in 
Table 2. Statistics of the count-rate and area limited complete subsample. The size of the areas is given as R.A. $\times$ DEC. $<T_{\mathrm{int}}>$ is the median integration time in the respective study area. " $C R$ limit" denotes the adopted count-rate limit. The median values of the column density of neutral hydrogen, $\left\langle N_{\mathrm{H}}\right\rangle$, for the individual areas are from Dickey \& Lockman (1990). SIM/NED: Identifications based exclusively on cross-correlation with SIMBAD and NED data bases, respectively, i.e. for these sources no new observations were obtained

\begin{tabular}{|c|c|c|c|c|c|c|c|}
\hline \multicolumn{8}{|c|}{ study area } \\
\hline & I & II & III & IVac & $\mathrm{Va}$ & VI & total \\
\hline Size $\left[{ }^{0} \times{ }^{\circ}\right]$ & $12 \times 12$ & $12 \times 12$ & $12 \times 12$ & $6 \times 12$ & $6.2 \times 6$ & $12 \times 12$ & $685.2^{\square}$ \\
\hline$C R$ limit $\left[\mathrm{ct} \mathrm{s}^{-1}\right]$ & 0.03 & 0.03 & 0.03 & 0.03 & 0.01 & 0.03 & \\
\hline$<N_{\mathrm{H}}>\left[10^{20} \mathrm{~cm}^{-2}\right]$ & 11.5 & 4.4 & 4.0 & 1.6 & 3.9 & 5.5 & \\
\hline$<T_{\mathrm{int}}>[\mathrm{s}]$ & 350 & 420 & 420 & 490 & 2300 & 320 & \\
\hline No. of sources: & 100 & 122 & 124 & 110 & 125 & 93 & 674 \\
\hline Observed: & 93 & 101 & 112 & 102 & 118 & 89 & 615 \\
\hline SIM/NED id. & 7 & 21 & 12 & 8 & 7 & 4 & 59 \\
\hline total & 100 & 122 & 124 & 110 & 125 & 93 & 674 \\
\hline
\end{tabular}

Cananea, which we used for the optical identification, it is not possible to obtain spectra with sufficient $\mathrm{S} / \mathrm{N}$ to identify reliably the featureless spectrum of a BL Lac fainter than about 19th magnitude (see Sect. 3). For the identification of these faint BL Lac objects we therefore use additionally their characteristic intrinsic optical variability (see below).

Table 3. Estimated X-ray flux limits in the 0.1 to $2.4 \mathrm{keV}$ energy band (in $10^{-13} \mathrm{erg} \mathrm{s}^{-1} \mathrm{~cm}^{-2}$ ) for coronal emitters, $f_{\mathrm{x}}^{\text {cor }}$, AGN, $f_{\mathrm{x}}^{\text {AGN }}$, clusters of galaxies, $f_{\mathrm{x}}^{\mathrm{cl}}$, and normal galaxies, $f_{\mathrm{x}}^{\text {gal }}$, in the individual study areas. Count rates $C R$ are given in units of $\operatorname{cts~s}^{-1}$. The fluxes depend on the intrinsic spectral energy distribution and on $N_{\mathrm{H}}$ (given in $10^{20} \mathrm{~cm}^{-2}$ ). For AGN a power law with a photon index of $\Gamma=2.0$ was used. For normal galaxies (ellipticals) and clusters of galaxies we assumed a spectrum of thermal bremsstrahlung with temperatures of $\log T=7.1$ and 8 , respectively

\begin{tabular}{llrlrll}
\hline area & $C R$ & $N_{\mathrm{H}}$ & $f_{\mathrm{x}}^{\text {cor }}$ & \multicolumn{1}{c}{$f_{\mathrm{x}}^{\text {AGN }}$} & $f_{\mathrm{x}}^{\text {cl }}$ & $f_{\mathrm{x}}^{\text {gal }}$ \\
\hline I & $\geq 0.03$ & 11.5 & 1.8 & 10.2 & 7.1 & 8.9 \\
II & $\geq 0.03$ & 4.4 & 1.8 & 6.9 & 5.5 & 6.1 \\
III & $\geq 0.03$ & 4.0 & 1.8 & 6.6 & 5.4 & 5.9 \\
IVac & $\geq 0.03$ & 1.6 & 1.8 & 4.1 & 4.2 & 3.8 \\
Va & $\geq 0.01$ & 3.9 & 0.6 & 2.2 & 1.8 & 1.9 \\
VI & $\geq 0.03$ & 5.5 & 1.8 & 7.7 & 5.8 & 6.7 \\
\hline
\end{tabular}

The flux limits given in Table 3 for clusters of galaxies correspond to visual magnitudes of the brightest galaxies in the clusters of about $V \lesssim 21^{\mathrm{m}}$ in area Va, and $\lesssim 20.5^{\mathrm{m}}$ in all other areas. Assuming a Schechter cluster luminosity function (Schechter 1976) the limiting magnitude of $23^{\mathrm{m}}$ in the direct imaging mode (see below) thus allows the detection of distant clusters of galaxies contained in our flux-limited subsample.
Normal elliptical galaxies are expected to be brighter than about $V \approx 18^{\mathrm{m}}$ in area Va, and brighter than about $17^{\mathrm{m}}$ in the other areas.

These estimates show that with the exception of faint BL Lacs the different classes of X-ray emitters are within the range of sensitivity of the instrumentation we used for the optical identification and which is described in the following section.

\section{Optical observations}

For all X-ray positions in our sample, with the exception of a few positions for which obvious optical counterparts were known from SIMBAD, NED or other astronomical data bases (see below), we obtained optical images and spectra. These observations were carried out at the $2.15 \mathrm{~m}$ telescope of the Guillermo Haro Observatory which is located near Cananea, Sonora, Mexico and which is operated by INAOE. For this purpose a dedicated focal reducer camera, the Landessternwarte Faint Object Spectrograph and Camera $(L F O S C)$ was constructed at the LSW. The instrument was attached to the Cassegrain focus of the telescope and allows direct CCD imaging, filter photometry, and multi-object spectroscopy. It is equipped with an EEV P8603 CCD detector $(385 \times 578$ pixels, $22 \mu \mathrm{m}$ linear pixel size). With a $10^{\prime} \times 6^{\prime}$ field of view the image scale is $1^{\prime \prime}$ pixel $^{-1}$.

For direct imaging we used Johnson $B$ and Cousins $R$ filters (Bessell 1979). In this observing mode a limiting magnitude of $\approx 23^{\mathrm{m}}$ can be reached within $900^{\mathrm{s}}$. Observations in $B$ and $R$ were used to derive the colours for a rough first object classification.

For the spectroscopic observations we used masks with circular holes of $3^{\prime \prime}$ projected diameter in the focal plane of the telescope. These masks were produced directly from the CCD images using a computer controlled drilling device. In addition to the object holes each mask contains holes producing spectra of the sky background near the objects. Alternatively, a slit mask can be inserted in order 
to perform normal long slit spectroscopy. The spectra are oriented in E-W direction. Perpendicular to the direction of dispersion, i.e. in N-S direction, a minimum separation of the holes of at least $5^{\prime \prime}$ was found to be necessary to avoid overlapping spectra. Therefore more than one spectral exposure was required if several candidate objects separated by less than $5^{\prime \prime}$ in N-S direction were present in the field. Figure 4 shows an example for such a field.

Two different grisms giving reciprocal linear dispersions of $250 \AA \mathrm{mm}^{-1}$ and $360 \AA \mathrm{mm}^{-1}$, respectively, were used. For holes 2.5 to the east of the field centre (which is also the position of the long slit) the spectral intervals covered were $4000-7200 \AA$ and $4200-8800 \AA$, respectively. With a hole diameter of $3^{\prime \prime}$ the spectral resolution $\Delta \lambda$ was about $13 \AA$ and $18 \AA$, respectively, i.e. a resolving power of $R=\lambda / \Delta \lambda \approx 400$ at central wavelengths.

For flat fielding and wavelength calibration of the spectra built-in halogen, and neon and xenon lamps were used. Flux standard stars were observed for flux calibration of the spectra. The spectra were reduced with a ESO-MIDAS based software package available at the Landessternwarte.

Accurate sky subtraction was achieved by using the intensity of the night sky emission line of $[\mathrm{OI}] \lambda 5577$ for calibrating small differences in the throughput of the individual mask holes.

For the classification of stellar counterparts we observed a grid of spectroscopic standard stars with spectral types between $\mathrm{O}$ and late $\mathrm{M}$, and luminosity classes I, III, and V using both grisms. Tests showed that with these standard stars a spectral classification with an accuracy of better than 5 subclasses can be obtained for objects brighter than about $V \approx 19^{\mathrm{m}}$ with $40^{\mathrm{m}}$ exposure time. Therefore, even the optically faintest $\mathrm{X}$-ray luminous coronal emitters expected in our subsample could be identified with $L F O S C$. Counterparts with emission lines, as e.g. AGN, could be identified as faint as $V \approx 20^{\mathrm{m}}$ to $21^{\mathrm{m}}$.

Examples of observations obtained with $L F O S C$ are shown in Figs. 3, 4, and 5. Usually possible counterparts within a circle of about $50^{\prime \prime}$ to $60^{\prime \prime}$ radius around the X-ray position were observed (see below). This is typically the $3 \sigma$ error circle as calculated by the standard ROSAT reduction software (SASS) (see Paper I). The average SASS $90 \%$ error circles which are indicated in the figures are of the order of $40^{\prime \prime}$ to $45^{\prime \prime}$. As noted below, our identifications discussed in Sect. 5 showed that the true error circles are, in fact, smaller.

The observations collected in Cananea were supplemented by spectroscopic observations with the $2.2 \mathrm{~m}$ telescope at ESO, La Silla, and with the $72 \mathrm{~cm}$ Waltz telescope at the Landessternwarte Heidelberg. In March 1996 we observed part of the sources in area III at the ESO/MPIA $2.2 \mathrm{~m}$ telescope. These observations were obtained with the EFOSC2 spectrometer which was equipped with a Thomson $1024 \times 1024$ pixel CCD chip (ESO CCD \#19). The spectral resolution obtained with grism \#1 and the
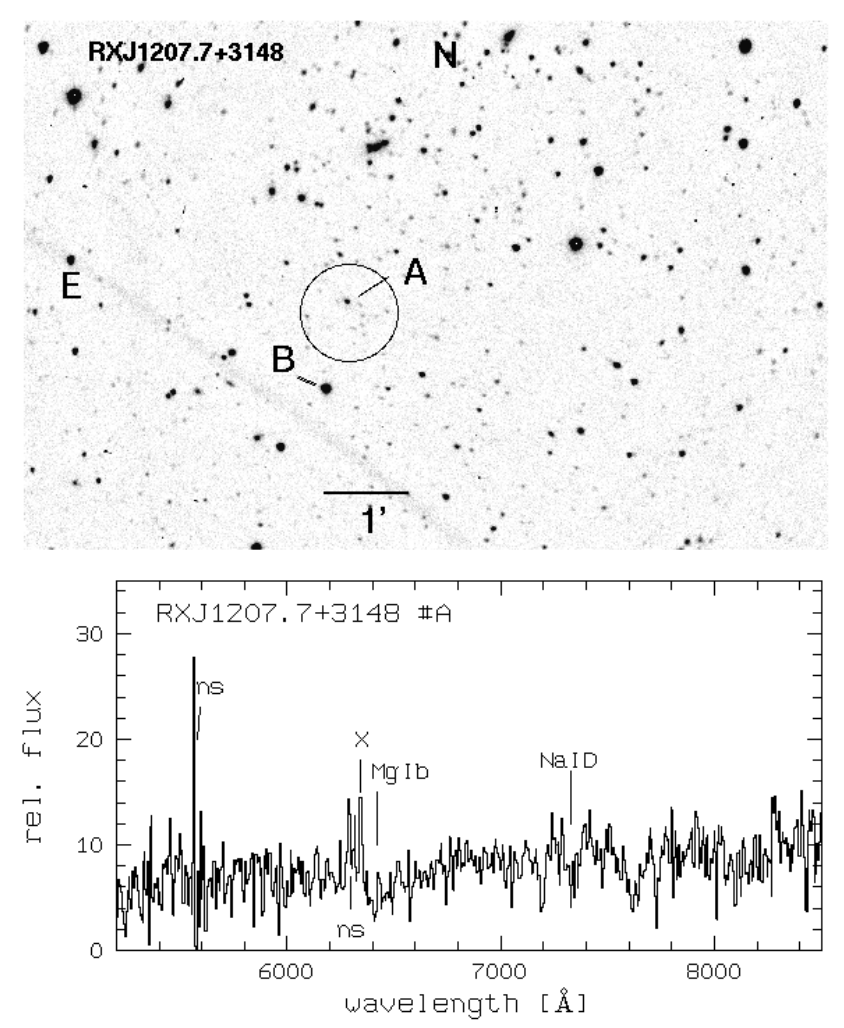

Fig. 3. $R$ band image of the field around the X-ray source RXJ1207.7+3148. The $90 \%$ SASS error circle (radius $35^{\prime \prime}$ ) is indicated. Many faint and diffuse objects, most likely distant galaxies, are visible. The brightness of the faintest visible objects is $R \approx 23$. For the two brightest objects near the source position, designated by "A" and "B", spectra were obtained. The remaining objects are too faint for spectroscopy. Object "A", whose spectrum is shown in the lower panel, appears to be a galaxy with $R \approx 20.3^{\mathrm{m}}$ and $B \approx 22.3^{\mathrm{m}}$. Absorption features most likely due to $\mathrm{Mg} \mathrm{Ib}$ and $\mathrm{Na} \mathrm{ID}$ with a redshift of $z \approx 0.23$ are visible. Residuals of the night sky lines are marked by "ns", " $\mathrm{x}$ " is a cosmic. Object " $\mathrm{B}$ " is a 16th magnitude F-type star. This star is visually too faint to be a plausible counterpart. On the $B$ image no object brighter than about $22^{\mathrm{m}}$ was visible making a QSO as counterpart unlikely. The galaxy "A" is visually too faint to be a plausible counterpart of the X-ray source (see Sect. 4.3). Hence, the most likely identification of this X-ray source is a distant cluster of galaxies

$1^{\prime \prime}$ slit (cf. ESO Users Manual) was $\sim 24 \AA$ and hence comparable to that achieved with the lower resolution grism of $L F O S C$. A sample of bright stellar counterparts previously identified with $L F O S C$ were observed with higher spectral resolution between March and October 1993 at the $72 \mathrm{~cm}$ Waltz telescope in order to study the spectra in more detail. A Boller \& Chivens spectrograph attached to the Nasmyth focus and equipped with an EEV P8603 CCD chip with $385 \times 57822 \mu \mathrm{m}$ pixels was used. The grating with 1200 lines $\mathrm{mm}^{-1}$ yielded a reciprocal linear dispersion of $44 \AA \mathrm{mm}^{-1}$, and (with a $2.4^{\prime \prime}$ slit) a spectral 

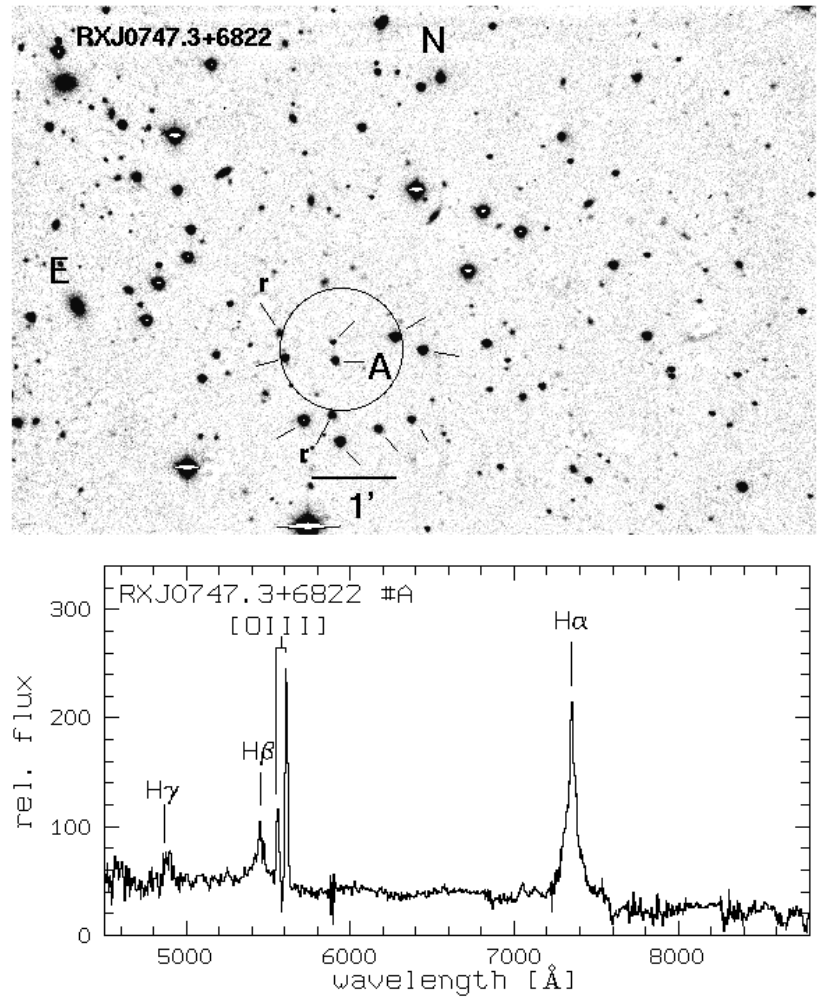

Fig. 4. $R$ image of the X-ray source RXJ0747.3+6822. The $90 \%$ SASS error circle is indicated (radius $44^{\prime \prime}$ ). With two exposures spectra of all objects within $60^{\prime \prime}$ radius around the RASS position could be obtained. " $r$ " denotes reflex images of bright stars in the field. In the lower panel the spectrum of object "A" is displayed which is the likely counterpart of the RASS source. It is a Sy 1 galaxy with $V \approx 18^{\mathrm{m}}$ at redshift $z=0.120$

resolution of about $2 \AA$. The observed spectral region was $6250-6750 \AA$.

Because of varying weather conditions most of the direct images could not be directly calibrated photometrically. Therefore we started an additional observing program to obtain secondary photometric sequences for the fields around each X-ray source. These photometric observations are being carried out in Cananea and at the Calar Alto $1.23 \mathrm{~m}$ telescope in Spain. Details will be described elsewhere. At this time and throughout the present paper we use photometry taken from the HST Guide Star Catalog (Lasker et al. 1988) (GSC) and from the APM catalogue (Irwin \& McMahon 1992). For the APM photometric data $V$ magnitudes can be estimated from $O$ and $O-E$ by using the relation $O-E \approx 2(B-V)$ by (Irwin \& McMahon 1992) and the colour transformation determined for the POSS plates by Humphreys et al. (1991) who found $O-B$ to be nearly independent of $B-V$ to within $0.3^{\mathrm{m}}$ for $0<B-V<1.7$. Likewise, an estimate for $R$ can be obtained from $E$ and $O$.

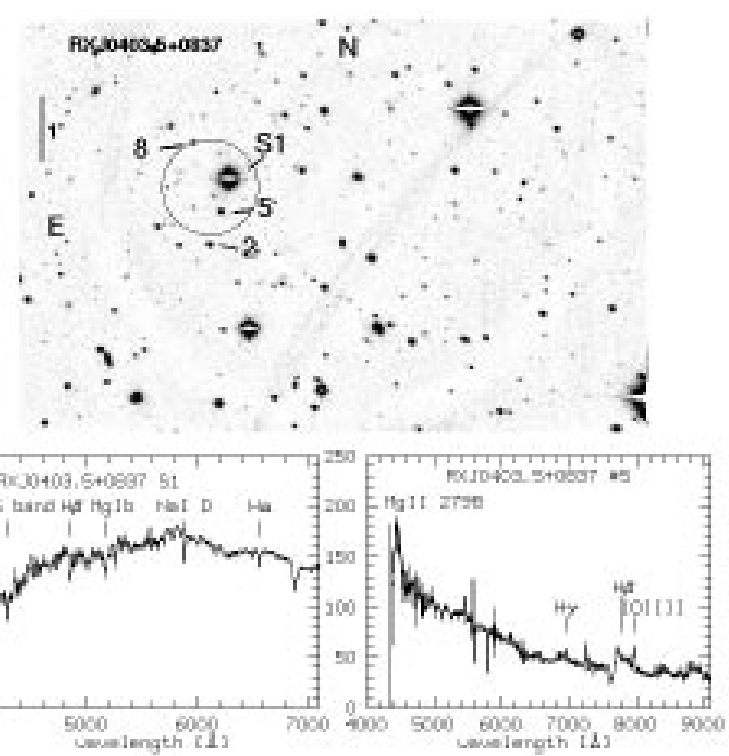

Fig. 5. $R$ image of the position of the $\mathrm{X}$-ray source RXJ0403.5+0837. In the the $90 \%$ SASS error circle (radius $44^{\prime \prime}$ ) several possible candidates for the optical counterpart are visible. The objects observed spectroscopically are designated by S1, "2", "5", and " 8 ". The bright object S1 is a 13th magnitude $\mathrm{G}$ to K-type star. Object \#5 with $V \approx 18.4^{\mathrm{m}}$ is a QSO with $\mathrm{H} \beta+\left[\mathrm{O}_{\mathrm{III}}\right]$ and $\mathrm{Mg} \mathrm{II} \lambda 2798$ at redshift $z=0.589 . \mathrm{H} \beta$ partly falls into the atmospheric band at $7600 \AA$. Each of the two objects could be the X-ray source or at least contribute to the observed X-ray flux (see text). The remaining objects are faint stars which can be excluded for being the counterpart of the X-ray source

Since Sep. 1990 we observed more than 800 RASS positions and we obtained in this way more than 3500 spectra of suspected optical counterparts. Meanwhile, observations or literature identifications (SIMBAD and NED databases) exist for nearly all sources in the count-rate and area limited complete subsample described above. In the next section we discuss the method of optical identification.

\section{Criteria for optical identification}

In earlier optical identification programs of X-ray sources very often possible candidates in the error box were observed until a "plausible" counterpart was found. For the identification of the EMSS X-ray sources Stocke et al. 1991) e.g. used a plausibility criterion based on the X-ray-to-optical flux ratio.

In the present investigation we used a different strategy. As described above our instrumental setup allows multi-object spectroscopy and hence offers the possibility to observe all candidates in the error circle with just one or two exposures. The candidate selection is limited by the sensitivity of the instrument. The X-ray-to-optical flux ratio, together with other $\mathrm{X}$-ray characteristics like 
hardness ratios and extension parameter, are used additionally only and as secondary criteria for the identification. In most cases an unambiguous identification of an object as optical counterpart of an X-ray source is possible with the CCD direct images and the spectra we observed.

Only in a few cases where known X-ray sources or obvious counterparts were found in the SIMBAD or NED databases at distances smaller than $30^{\prime \prime}$ from the X-ray source position no new observations were carried out. Most of these obvious objects are known QSOs, BL Lacs, or bright stars. If on the APM blue finding charts other potential candidates were visible, new observations were obtained. Abell clusters were usually reobserved in order to check if e.g. AGN are present in the error circle.

It has been repeatedly suggested, e.g. by Stocke et al. (1991), to select different object classes (such as e.g. extragalactic sources) on the basis of $f_{\mathrm{x}} / f_{V}$ prior to spectroscopic identification. In many cases this method leads to reliable pre-identifications. However, we found numerous cases where this quantity alone does not allow a decision whether an X-ray source is of extragalactic or stellar origin. An example is shown in Fig. 5 (RXJ0403.5+0837) for which an X-ray count rate of $0.05 \mathrm{cts} \mathrm{s}^{-1}$ was measured. In the error circle a star with $V \approx 13^{\mathrm{m}}$ and a fainter object with $V \approx 18^{\mathrm{m}}$ are present. Spectroscopy showed the star to be of type $\mathrm{G}$ to $\mathrm{K}$ and the fainter object to be a QSO. Both would have a $f_{\mathrm{x}} / f_{V}$ ratio suitable for their class. An identification in such a case requires additional higher resolution spectroscopic observations in order to check if the star shows signs of coronal activity. In this special case a further clue to the nature of the counterpart comes from the hardness ratio $H R 1$ which is +0.83 and therefore is more indicative for a QSO than for a coronally active star. Note, however, that certain classes of coronally active stars, like e.g. T Tauri stars, also can exhibit rather hard X-ray spectra.

\subsection{Stellar counterparts: Coronal emitters, binaries etc.}

Tracers for coronal activity in mid-to-late type stars are emission components in the CaII resonance lines and Balmer line emission. Both spectroscopic characteristics are closely related to coronal X-ray activity (e.g. Fleming et al. 1995) and hence are an ideal tool for the identification of coronally active sources as optical counterparts of $\mathrm{X}$-ray sources. Since $L F O S C$ uses inexpensive glass optics and a "thick" CCD, observations of the spectral region below $\approx 4000 \AA$ were not possible. Moreover, our spectral resolution would not have been sufficient to detect emission components in the absorption troughs of Ca II. For our identification procedure we were therefore restricted to the use of Balmer emission components.

Among the stellar counterparts the coronally active $M$ dwarfs represent a group of objects which can be reliably identified with our low resolution spectra due to their molecular absorption bands and strong $\mathrm{H} \alpha$ emission lines. Occasionally also $\mathrm{H} \beta$ is visible in emission. In $\mathrm{K}$ and G type stars very often the absorption strength of $\mathrm{H} \alpha$ is weaker than expected for the respective spectral type, indicating that the photospheric absorption line is filled in by chromospheric emission.

In several cases, however, with our low-resolution spectra we are not able to find direct spectroscopic indicators for chromospheric activitity. This is particularly the case for $\mathrm{F}$ to $\mathrm{G}$ type stars, among them also X-ray active binary systems e.g. of the RS CVn class. In most of these cases we observed all possible counterparts in the ROSAT error circle in order to search, e.g., for fainter AGN. Exceptions are a few very bright stars which do not allow to detect much fainter objects in their immediate vicinity. Occasionally, the presence of a distant cluster of galaxies in the field of a star near the X-ray position was indicated in the deep $R$ image. If no other potential counterpart than the star was found and if the X-ray properties were in agreement with the average class characteristics the star was considered to be the X-ray emitter. In these cases the identification should be regarded as tentative requiring further higher resolution spectroscopic observations of the stellar object.

Although our sample contains sources at high galactic latitude the presence of young objects cannot be excluded as shown by Favata et al. (1993). In fact, in the small sample of stellar counterparts observed at the $72 \mathrm{~cm}$ telescope of the Landessternwarte (see Sect. 3) we found $\operatorname{Li} I \lambda 6708$ absorption characteristic for pre-main sequence X-ray sources in several objects (Ziegler 1993). These sources are listed in Table 4. A more detailed study of stellar counterparts is under way in order to search for binarity, for Ca II emission, for Li I $\lambda 6708$ absorption and to determine the stellar rotational velocity (Metanomski et al. 1996).

Table 4. Stellar counterparts with strong Li I $\lambda 6708$ absorption line. $W(\mathrm{Li})$ : equivalent width of $\operatorname{Li} \mathrm{I} \lambda 6708$ in $\mathrm{m} \AA$

\begin{tabular}{llll}
\hline ROSAT source & catalog name & spec. type & $W(\mathrm{Li})$ \\
\hline RXJ1103.9-0741 & BD -033040B & K1V & 160 \\
RXJ1231.0+3125 & SAO 63037 & F8V & 140 \\
RXJ1251.6+2732 & SAO 82537 & G0V & 140 \\
RXJ1625.5+7123 & SAO 8513 & G2IV & 120 \\
RXJ1626.4+7203 & SAO 8520 & K2III & 540 \\
RXJ1747.6+7307 & SAO 8927 & F9IV & 120 \\
RXJ1818.5+7042 & SAO 9067 & G5V & 230 \\
RXJ1818.5+7042 & SAO 9069 & K2V & 190 \\
RXJ1825.8+7144 & SAO 9124 & F5V & 100 \\
RXJ1839.0+7257 & SAO 9189 & F9IV & 110 \\
RXJ1857.9+7703 & SAO 9296 & F2V & 100 \\
\hline
\end{tabular}

\subsection{Active galactic nuclei}

The spectroscopic identification of most AGN (i.e. Seyfert galaxies, QSOs, and LINER; for BL Lac objects see 
below), is based on the detection of emission lines. With the low resolution grism $L F O S C$ allows the detection of the typical lines of $\mathrm{H} \beta+[\mathrm{OIII}]$ and $\mathrm{H} \alpha$ for redshifts below about 0.3 , the exact range of $z$ depending on the position of the holes (see Sect. 3). At higher redshifts up to $z \approx 0.7 \mathrm{H} \beta+[\mathrm{O} \mathrm{III}]$ are contained in the observed wavelength range. At $z \gtrsim 0.5$ the emission feature of Mg II $\lambda 2798$ becomes detectable at the blue edge of the spectra. At even higher redshifts the emission features of e.g. Mg II $\lambda 2798$ and $\mathrm{C}$ III] $\lambda 1909$ are contained in the observed range. At $z \gtrsim 2 \mathrm{Mg}$ II $\lambda 2798$ disappears from the observed wavelength range, however, C III] $\lambda 1909 \mathrm{C}$ IV $\lambda 1550$ are visible. At $z \gtrsim 2.3 \mathrm{Ly} \alpha$ becomes detectable at the short wavelength limit. Hence for a wide range of redshifts one or more typical AGN emission feature is contained in the wavelength range of the classification spectra obtained with the lower resolution grism of LFOSC.

Most of the objects identified as AGN show broad emission lines of $\mathrm{H} \alpha$ or $\mathrm{H} \beta$. In a few cases $\mathrm{H} \alpha$ is not in the observed wavelength range and $\mathrm{H} \beta$ is too weak to measure the line width although $\mathrm{H} \beta$ and [O III] are obviously present. In these cases the AGN type cannot be established from our data.

\subsection{Individual galaxies and clusters of galaxies}

The identification of individual non-AGN type galaxies as $\mathrm{X}$-ray emitters is sometimes ambiguous. Generally, normal galaxies are not expected to be strong X-ray emitters. As discussed e.g. by Fabbiano et al. (1992), Peace \& Sansom (1996) and Mackie et al. (1996) X-ray luminosities for spirals are of the order of $L_{\mathrm{x}} \approx 10^{38} \mathrm{erg} \mathrm{s}^{-1}$ to less than $10^{42} \mathrm{erg} \mathrm{s}^{-1}$, elliptical and S0 galaxies are more luminous with $L_{\mathrm{x}} \approx 10^{39}$ to $10^{43} \mathrm{erg} \mathrm{s}^{-1}$. Thus the most luminous normal galaxies are at least one order of magnitude less luminous than AGN and therefore only nearby galaxies or the most luminous $\mathrm{E}$ and $\mathrm{S} 0$ type galaxies are expected to be detectable in the X-ray band.

Most of the galaxies found at ROSAT positions seem to be members of groups or even of clusters of galaxies and hence it is not clear whether the X-ray emission is caused by an individual galaxy or whether extended gas in the group or cluster is the origin of the the $\mathrm{X}$-ray emission. Occasionally the ROSAT extension parameter indicates extended emission suggesting that the $\mathrm{X}$-ray emission is produced by the hot IGM gas distributed between the galaxies of a cluster or group. However, in normal galaxies the sources producing the X-ray emission are either spatially distributed individual sources or extended halos of hot gas. Therefore the extension parameter by itself is not a sufficient criterion for the identification of galaxy clusters. In cases of galaxies present at the position of extended X-ray emission we identified the X-ray source as individual galaxy only if the emission is well-centred on the candidate galaxy, if no neighbouring galaxies with similar redshifts are visible nearby, and if the X-ray luminosity is not too high for a normal individual galaxy.

Galaxy clusters were detected by the redshifts of its galaxy members or, when the galaxies were too faint, by the gradient of the number density of objects on the CCD direct image and/or by the distribution of the luminosities of its galaxy members, the cluster luminosity function.

The magnitude of the central galaxy of the faintest possible cluster counterpart is about $V \sim 21^{\mathrm{m}}$ (see Sect. 2.2). Assuming a Schechter luminosity function (Schechter 1976) the tenth brightest galaxy member should be less than a magnitude fainter, that is, easily detectable with direct imaging. Assuming an absolute magnitude of the brightest member of $M_{V} \sim-23.5$ (Sarazin 1988) and a Hubble constant of $H=75 \mathrm{~km} \mathrm{~s}^{-1} \mathrm{Mpc}^{-1}$ the Abell radius of such a cluster is $\sim 50^{\prime \prime}$, and even the faintest clusters are therefore resolvable as such.

The cluster luminosity function can be estimated if the cluster radius is smaller than the size of our CCD image frame of $6^{\prime} \times 10^{\prime}$ such that the background source distribution can be roughly subtracted. This is the case for an Abel radius smaller than about 3'. (A gradient in the number distribution might be detected even up to an Abel radius of $6^{\prime}$ ). X-ray emission of clusters is caused by hot virialized gas which traces the cluster potential. Therefore the center of the cluster and with it the highest projected number density should coincide with the center of the X-ray emission. If this was the case we identified the X-ray source as a galaxy cluster.

When the direct image showed indications for the existence of a galaxy cluster in the field of view, spectra of posible member galaxies were taken even outside the ROSAT positional error circle. A detailed study of these sources was carried out by Kneer (1996) and will be discussed in a forthcoming paper. A spectroscopic detection is possible if about the five brightest cluster members are brighter than our magnitude limit for spectroscopy $\left(V_{\min }\right.$ $=19^{\mathrm{m}}$ ) and if they are within our field of view. Since the fifth brightest member of a cluster has typically an absolute magnitude of about $M_{V}=-23^{\mathrm{m}}$ (Sarazin 1988) we can detect a cluster spectroscopically up to a distance of $2500 \mathrm{Mpc}$, i.e. a redshift of 0.63 , when assuming a Hubble constant of $75 \mathrm{~km} \mathrm{~s}^{-1} \mathrm{Mpc}^{-1}$. This corresponds to an Abell radius of $2.7^{\prime}$. Fainter, that is more distant clusters will have a smaller cluster radius so that a gradient in the number distribution of objects inside our CCD image frame of $6^{\prime} \times 10^{\prime}$ will be visible and they can be detected as described above.

When the Abel radius of a cluster becomes larger than our image frame $\left(R_{\mathrm{A}}>3^{\prime}\right)$, that is, at a cluster red shift of about 0.57 , the gradient of the galaxy number distribution and therefore the exact center of the galaxy cluster becomes more difficult to be determined. There should however still be sufficiently many galaxies in the field of view to detect the presence of a cluster spectroscopically up to an Abel radius of $\left(R_{\mathrm{A}} \sim 10^{\prime}\right)$, corresponding to 
a redshift of 0.17 . All closer clusters should be very well known and can be identified with the help of literature search using the SIMBAD or NED data base.

A few of the observed galaxies could be cooling flow galaxies as defined by Stocke et al. (1991). Some of them are obviously members of clusters. They show narrow emission of $\mathrm{H} \alpha$, often also [SII], but no strong [O III]. $[\mathrm{OII}]$ is usually stronger than [O III] in this class of objects. Because candidates are normally at small redshifts the emission lines of $[\mathrm{O} \mathrm{II}]$ are either not in the observed wavelength region or often not discernible due to the noise in the blue part of the spectra. For these objects spectra in the blue wavelength region are required to confirm the tentative classification as cooling flow galaxies. In our catalog these sources are preliminarily classified as cluster of galaxies.

\subsection{BL Lac objects}

A necessary criterion for the classification of a candidate as BL Lac object is a featureless continuum without emission lines. In order to discriminate BL Lacs against normal elliptical galaxies Stocke et al. (1991) defined a criterion to measure the contribution of stellar light by making use of the Ca II break. Since our spectra usually have a very low $\mathrm{S} / \mathrm{N}$ in the blue spectral region this criterion cannot be used for our spectroscopic data. However, the absence of $\mathrm{Mg} I \mathrm{~b}$ and of Na I D absorption features which are located further in the red, and the absence of Balmer absorption lines for blue objects at least allow to distinguish the visually brighter $\left(V \lesssim 18^{\mathrm{m}}\right)$ BL Lac candidates from stars.

For the identification of fainter BL Lacs, which we cannot classify directly spectroscopically, we started a photometric monitoring program of the potential candidates searching for the intrinsic variability of BL Lacs. For this purpose we obtain deep $R$ images of the fields around the candidates. As additional criteria for the fainter candidates found by means of the optical variability we use X-ray-to-optical flux ratio and the location in the $\alpha_{\mathrm{ox}}-\alpha_{\text {ro }}$ diagram. The use of these parameters for the identification of BL Lacs was recently discussed by Nass et al. (1996). The $\alpha$ parameters are defined as $\alpha_{\mathrm{ox}}=-\left(\log S_{\mathrm{x}} / S_{2500}\right) / 2.605$, and $\alpha_{\mathrm{ro}}=$ $\left(\log S_{5 \mathrm{GHz}} / S_{2500}\right) / 5.38$, where $S_{\mathrm{x}}, S_{2500}$, and $S_{5 \mathrm{GHz}}$ are the monochromatic fluxes per frequency interval at $2 \mathrm{keV}$, $2500 \AA$, and at $5 \mathrm{GHz}$ (cf. e.g. Tananbaum et al. 1979). Radio fluxes were taken from the $4.85 \mathrm{GHz}$ survey of Gregory \& Condon (1991). For those objects for which no radio measurement exists we adopted an upper limit for the flux at $4.85 \mathrm{GHz}$ of $20 \mathrm{mJy}$. As mentioned in Sect. 3 the photometric calibration is still being improved. At this time we therefore use photometry from the APM (see Sect. 3) catalog for the calculation of the flux ratios and the continuum slopes. For classification purposes the accuracy is acceptable since an error of $1^{\mathrm{m}}$ in $V$ will introduce an uncertainty of the $\log f_{\mathrm{x}} / f_{V}$ ratio of the order of
0.4. Hence stars and BL Lacs can still be distinguished. Likewise, the corresponding uncertainties of the $\alpha$ coefficients are also acceptably small. Variable candidates without emission lines in their spectra are very likely BL Lac objects. Sources for which light curves are not yet available were classified as "possible BL Lacs" if a) no other "plausible" counterpart is present and b) objects without emission lines and suitable $\log f_{\mathrm{x}} / f_{V}$ and $\alpha$ parameters are present in the error circle.

\section{Results}

In this section we describe some first results, focussing mainly on areas I, IVac, and Va. These areas are of particular interest because they represent the extreme values of $N_{\mathrm{H}}$ and of the RASS integration time in our sample. Several identifications, in particular of stellar sources at low X-ray count rates, require further confirmation. These observations are still in progress. The full set of identifications will be published in Paper III.

\subsection{Total subsample of areas I, IVac, and Va}

The results based on our preliminary identifications for these three areas are summarized in Table 5. For 323 of the 335 sources of the subsample identifications exist, although some of them are still tentative or uncertain. These uncertain identifications will, however, not affect the following conclusions.

With 116 identifications the stellar counterparts represent a fraction of $\approx 35 \%$ of the objects. This is significantly higher than the fraction of stellar identifications in the EMSS by Stocke et al. (1991) who found only $26 \%$ stellar sources, but less than the $65 \%$ stellar counterparts in the EXOSAT HGLS Giommi et al. 1991). Note that $37 \mathrm{Ke} / \mathrm{Me}$ stars are among the stellar sources we identified. Surprisingly, also 3 bright A-type stars were found very close to the positions of X-ray sources in area I. In these cases the X-ray emission is attributed to unseen companions. Two of them exhibit hard X-ray spectra. Three white dwarfs, including one previously known (WD04601 ), were found. Their X-ray spectra are extremely soft with hardness ratios HR $1 \approx-1$. Furthermore three cataclysmic variables were identified.

The percentage of the individual object classes on the total identifications is clearly varying between the different areas. The NGP field IVac shows e.g. a much larger fraction of AGN and a smaller fraction of stars than area I. Since most stellar sources are at relatively small distances, this effect cannot be explained by the different lines of sight through the galactic stellar disk alone. This suggests that the different neutral hydrogen column densities $N_{\mathrm{H}}$ play an important role. Areas I and IVac represent the extreme cases of $N_{\mathrm{H}}$ with the largest column density in area I and the smallest in area IVac (see Table 2). With $80 \%$ the extragalactic sources are the dominant constituent in 
Table 5. Identifications for areas I, IVac, and Va. SIM/NED: Identifications based exclusively on the cross-correlation with the SIMBAD and NED data bases, respectively. "stars" includes normal stars, Ke, Me stars, CVs, binaries, and white dwarfs

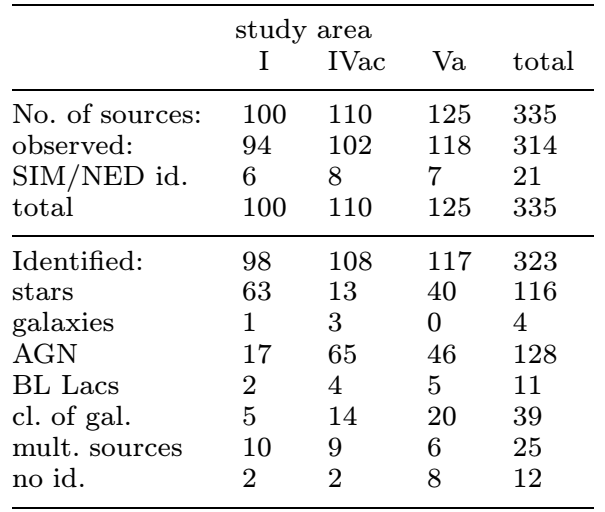

area IVac which is similar to the results of Hasinger et al. (1993) for the Lockman Hole. In area I only about $25 \%$ of the source are of extragalactic nature. With $63 \%$ the stellar sources are the dominant part in this area, in contrast to $12 \%$ in area IVac. Area V is intermediate, both with respect to the ratio of stellar to extragalactic sources and to $N_{\mathrm{H}}$. With $<H R 1>\approx+0.8$ the average hardness ratio of AGN in area $I$ is harder than that in area IVac which is $\langle H R 1>\approx 0.0$. Area $\mathrm{Va}$ is intermediate with $<H R 1>\approx+0.3$. This effect is clearly a consequence of the different $N_{\mathrm{H}}$ values. Likewise, the median redshifts of AGN, $z_{\text {med }}$, are different: 0.15 in area I and 0.32 in area IVac. Although in area Va with $z_{\text {med }}=0.29$ the median redshift is not significantly different from area IVac, we found AGN at higher redshift than in area IVac. This is probably a consequence of the greater sensitivity due to the longer integration times in area Va compensating the higher absorption and allowing the detection of more distant and hence X-ray fainter objects.

A small fraction of sources were preliminarily classified as "multiple" (see Table 5). This identification does not necessarily mean that more than one object is responsible for the X-ray emission. It rather denotes source positions for which our presently available spectra do not allow to determine a single obvious counterpart for the $\mathrm{X}$-ray source. The presence of two possible stellar counterparts represents the largest fraction, $\sim 40 \%$. Nearly all of these sources are located in area I which has the highest contribution of stellar counterparts. In five cases we found two AGN close to the X-ray position, in one of these even a third AGN exists which could also contribute to the $\mathrm{X}$-ray emission. We found several cases in which a known BL Lac object is located in (or projected on) a cluster of galaxies, as e.g. RXJ0416.8+0105 = H0414+009 in area
I. These objects do not appear in Table 5 as "multiple" object but were classified as BL Lacs.

\subsection{Positional uncertainty}

In the following we will make use of the identifications in the three areas to derive an estimate for the error of the X-ray positions. As mentioned in Sect. 3, we observed candidates in a circle with $50^{\prime \prime}$ to $60^{\prime \prime}$ radius which is approximately the $3 \sigma$ error circle calculated by the SASS. The positions of the optical counterparts were taken from the APM catalog in most cases. Positions of stars brighter than $6.5^{\mathrm{m}}$ are from Hoffleit (1964).

Clusters of galaxies, identifications with more than one plausible counterpart, and sources for which the identification is uncertain were excluded. Also excluded are a few sources for which due to image blending on the POSS plate no accurate optical position is available. In Fig. 6 histograms with the positional uncertainties derived from our identifications for the remaining sample of 231 sources are displayed. We plotted the differences of the positions of the X-ray sources, $\mathrm{X}$, and the optical counterparts, $\mathrm{CP}$, for declination and right ascension separately, i.e. $\triangle \mathrm{RA}(\mathrm{X}-\mathrm{CP})$ and $\triangle \mathrm{DEC}(\mathrm{X}-\mathrm{CP})$, respectively. Assuming a Gaussian distribution which is suggested by a Kolmogorov-Smirnov test on a 95\% significance level we derived a $1 \sigma$ error of $\approx 9^{\prime \prime}$ radius in $\mathrm{RA}$ and in DEC each. A small offset between optical and X-ray position in R.A. of $1^{\prime \prime}$ to $2^{\prime \prime}$ to the East was found. This is due to the motion of the satellite during the read-out time of the data (cf. Paper I).

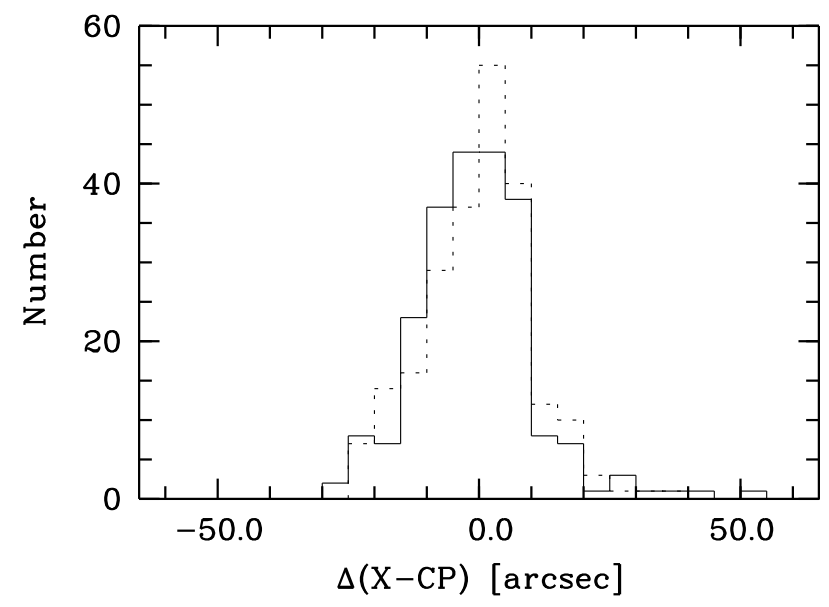

Fig. 6. Histograms of the positional uncertainties for sources in areas I, IVac, and Va. The differences in arcsec between X-ray position and optical counterpart position are plotted separately for RA (solid line) and DEC (dashed line). $1 \sigma$, i.e. the $67 \%$ error circle, is $\approx 9^{\prime \prime} .90 \%$ of the counterparts are found within $17^{\prime \prime}$ from the X-ray position in RA as well as in DEC 


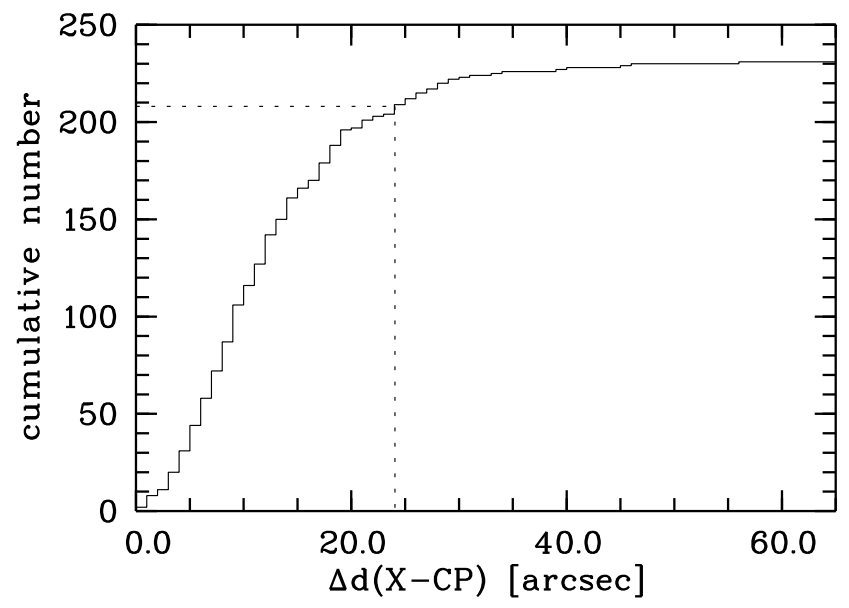

Fig. 7. Cumulative distribution of the distances between X-ray and optical source position. The resulting $90 \%$ error circle is $24^{\prime \prime}$, and the corresponding $1 \sigma$ uncertainty is $15^{\prime \prime}$

The cumulative number of counterparts within a given distance from the X-ray position is plotted in Fig. 7. From this we determined a $1 \sigma$ uncertainty of the distance between X-ray and optical position of $15^{\prime \prime}$ and a $90 \%$ error circle of $24^{\prime \prime}$ (see Fig. 7). The error circle is slightly larger if only sources with a total number of counts of less than 50 corresponding to typical count rates of $\leq 0.1 \mathrm{cts} \mathrm{s}^{-1}$ in area I and IVac and $\leq 0.03 \mathrm{cts} \mathrm{s}^{-1}$ in area Va are considered. For these sources we measured a $90 \%$ error circle of $27^{\prime \prime}$. Our estimates for the error circle are, therefore, significantly smaller than those calculated by the SASS (cf. Paper I) which yielded $90 \%$ error circles on the order of $40^{\prime \prime}$ to $45^{\prime \prime}$ (see above).

Acknowledgements. The ROSAT project is supported by the Bundesministerium für Bildung und Wissenschaft and the Max-Planck-Gesellschaft. We would like to thank the ROSAT team for observing and processing the ROSAT All-Sky Survey data. We also thank the observers who contributed to the optical observations: J. Alcala, C. Alvarez, H. Bock, H. Bravo, L. Corral, L. de la Cruz, U. Erkens, C. Fendt, Th. Gäng, J. Guichard, M. Kümmel, R. Madejski, A. Marquez, O. Martinez, A. Piceno, A. Porras, F. Ruzicka, Th. Szeifert, J. R. Valdes, F. Valera, G. Vazquez, R. Wichmann, and K. Wilke. We also would like to thank the staff of the Guillermo Haro Observatory for the support during the observations. This work was supported by DARA under grant Verbundforschung 50 OR 90017.

\section{References}

Bessell M.S., 1979, PASP 91, 589

Danziger J., et al. (in preparation)

Dickey J.M., Lockman F.J., 1990, ARA\&A 28, 215

Fabbiano G., Kim D.-W., Trinchieri G., 1992, ApJS 80, 531
Favata F., Barbera M., Micela G., Sciortino S., 1993, A\&A 277,428

Fleming T.A., Schmitt J.H.M.M., Giampa M.S., 1995, ApJ 450,401

Gioia I.M., Maccacaro T., Schild R.E., et al., 1990, ApJS 72, 567

Giommi P., Tagliaferri G., Beuermann K., et al., 1991, ApJ 378,77

Gregory P.L., Condon J.J., 1991, ApJS 75, 1011

Hasinger G.R. Burg R., Giacconi R., et al., 1993, A\&A 275, 1

Hasinger G.R., Burg R., Giacconi R., Hartner G., Schmidt M., Trümper J., Zamorani G., 1993, A\&A 291, 348

Hoffleit D., 1964, Yale catalogue of bright stars, Yale University, New Haven

Humphreys R.M., Landau R., Ghigo F.D., Zumach W., LaBonte A.E., 1991, AJ 102, 395

Irwin M., McMahon R., 1992, Newsletter of the Royal Greenwich Obs. No. 37

Kim D.-W., Fabbiano G., Trinchieri G., 1992, ApJ 393, 134

Kneer R., 1996, Ph.D. thesis, University of Heidelberg

Krautter J., Zickgraf F.-J., Thiering I., et al., 1996, A\&A (in preparation) (Paper IV)

Lasker B.M., Sturch C.R., Lopez C., et al., 1988, ApJS 68, 1

Mackie G., Fabbiano G., Harnden F.R., et al., 1996, in: Zimmermann H.U., Trümper J.E., Yorke H. (eds.), MPE report 263, "Röntgenstrahlung from the Universe". Garching, p. 385

Metanomski A., Krautter J., Pasquini L., 1996, in: Pallavicini R. and Dupree A. (eds.), ASP Conf. Ser., Cool stars, stellar systems and the sun (in press)

Motch C., Belloni T., Buckley D., et al., 1991, A\&A 246, L24

Nass P., Bade N., Kollgaard R.I., Laurent-Muehleisen S.A., Reimers D., Voges W., 1996, A\&A 309, 419

Peace G.A., Sansom A.E., 1996, in: Zimmermann H.U., Trümper J.E., Yorke H. (eds.), MPE report 263, "Röntgenstrahlung from the Universe". Garching, p. 387

Raymond J.C., Smith B.W., 1977, ApJS 35, 419

Read A., Ponman T., Strickland D., 1996, in: Zimmermann H.U., Trümper J.E., Yorke H. (eds.), MPE report 263, "Röntgenstrahlung from the Universe". Garching, p. 391

Sarazin C.L., 1988, "X-ray emission from clusters of galaxies". Cambridge University press, Cambridge, New York, New Rochelle, Melbourne, Sydney

Schechter P.L., 1976, ApJ 203, 297

Schmitt J.H.M.M., Fleming T.A., Giampa M.S., 1995, ApJ 450,392

Shanks T., Georgantopoulos I., Stewart G.C., et al., 1991, Nat 353,315

Stocke J.T., Morris S.L., Gioia I.M., et al., 1991, ApJS 76, 813

Tananbaum H., et al., 1979, ApJ 234, L9

Thiering I., Zickgraf F.-J., Krautter J., et al., 1996, A\&AS (in preparation) (Paper III)

Voges W., 1994, in: Haubold H.J. and Onuora L.I. (eds.) "Basic Space Science", p. 212

Voges W., Thiering I., Zickgraf F.-J., Krautter J., et al., 1996, A\&A (in preparation) (Paper I)

Ziegler B., 1993, Diploma thesis, University of Heidelberg 